Cambouis, la revue des sciences sociales aux mains sales

De la création de la

possibilité de l'enquête

à l'engagement

ethnographique :

entretien avec Olivier

Schwartz

Réalisé par Agnès Aubry, Morgane Kuehni \& Laure Scalambrin

\title{
Olivier Schwartz
}

Professeur émérite de sociologie, Université de Paris

o.schwartz@wanadoo.fr

\section{Agnès Aubry}

Membre du Centre de Recherche sur l'Action Politique de l'Université de Lausanne (CRAPUL), chercheuse invitée FNS au Graduate Center, City

University of New York (CUNY)

agnes.aubry@unil.ch

\section{Morgane Kuehni}

Professeure à la Haute école de travail social et de la santé de Lausanne HES.SO | HETSL

morgane.kuehni@hetsl.ch

\section{Laure Scalambrin}

Collaboratrice scientifique HES et boursière post-doctorat du domaine

Travail Social de la HES.SO, HES.SO | HETSL

laure.scalambrin@hetsl.ch

Date de publication : 30/06/2021

Dossier : Pratiques et politiques de la négociation pour accéder et se maintenir sur un terrain d'enquête

Comment citer : $10.52983 /$ crev.vi0.87

Licence: Cambouis publie ses contenus selon les termes de la Licence Creative Commons Attribution - Pas d'Utilisation Commerciale - Pas de Modification 4.0 International. Les auteurices gardent leurs droits de propriété intellectuelle pleine et entière sur leurs articles. 


\section{De la création de la possibilité de l'enquête à l'engagement ethnographique : entretien avec Olivier Schwartz}

\section{Réalisé par Agnès Aubry, Morgane Kuehni \& Laure Scalambrin}

\section{Olivier Schwartz}

Professeur émérite de sociologie, Université de Paris

o.schwartz@wanadoo.fr

\section{Agnès Aubry}

Membre du Centre de Recherche sur l'Action Politique de l'Université de

Lausanne (CRAPUL), chercheuse invitée FNS au Graduate Center, City

University of New York (CUNY)

agnes.aubry@unil.ch

\section{Morgane Kuehni}

Professeure à la Haute école de travail social et de la santé de Lausanne HES.SO | HETSL

morgane.kuehni@hetsl.ch

\section{Laure Scalambrin}

Collaboratrice scientifique HES et boursière post-doctorat du domaine Travail Social de la HES.SO, HES.SO | HETSL

laure.scalambrin@hetsl.ch

Nous rencontrons Olivier Schwartz le 25 juin 2019 à l'Université René Descartes-Paris 5 où il a achevé sa carrière d'enseignant en 2016. C'est un jour de canicule. Olivier Schwartz, avenant, a prévu des bouteilles d'eau pour chacune d'entre nous. Depuis son enquête au long court, publiée sous le titre Le monde privé des ouvriers, hommes et femme du Nord (Schwartz, 1990), puis son texte intitulé «L'empirisme irréductible », postfaçant Le Hobo, sociologie du sansabri (Schwartz, 1993), Olivier Schwartz est une figure incontournable de la sociologie française à double titre. Il a non seulement participé à renouveler en profondeur le débat sur les classes ouvrières, portant le regard dans la sphère privée, s'attachant à comprendre les conditions d'existence et modes de vie, mais il a aussi œuvré durant toute sa carrière à l'appropriation des méthodes ethnographiques par la sociologie et à leur diffusion. L'entretien réalisé avec Nicolas Renahy paru en 2016, intitulé « L'ethnographie comme expérience de conversion à la sociologie $»^{1}$, nous a convaincues de la pertinence et de l'intérêt de discuter avec Olivier Schwartz de ses pratiques d'enquête. Leur discussion est bien plus qu'un témoignage sur la trajectoire sociale et intellectuelle d'Olivier Schwartz, elle est aussi un « dialogue sur la nature même de l'ethnographie » (Renahy, 2016, p. 193). 
Notre démarche est moins ambitieuse du point de vue épistémologique et vise avant tout à revenir sur les aspects méthodologiques de deux grandes enquêtes menées par Olivier Schwartz : celle qu'il a conduite il y a une trentaine d'années sur la sphère familiale du monde ouvrier et celle qu'il mène aujourd'hui sur les conducteurs de la Régie autonome des transports parisiens (RATP). L'entretien reproduit ci-dessous met en lumière des pratiques fortement différenciées dans les deux grandes enquêtes précitées et permet de discuter au grand jour ce qui est souvent tu ou rendu invisible dans les restitutions et les publications, notamment en termes de négociation, au cœur de ce dossier de la revue Cambouis. Pour Olivier Schwartz, les méthodes sont toujours circonstanciées : s'il raconte volontiers la manière dont il mène ses enquêtes, il se refuse à livrer des « ficelles », pour reprendre le terme de Becker (2002). Soulignant que les règles déontologiques et la réflexivité sont essentielles à la posture ethnographique et irriguent toutes les étapes de ce type d'enquête, il partage ici, très humblement, les nombreux enseignements qu'il a pu en tirer et les dilemmes qui l'animent encore aujourd'hui.

Les thématiques abordées dans l'entretien sont multiples et s'entremêlent en partie. Nous pouvons néanmoins en dégager quelques-unes. Après avoir exposé sa manière de comprendre le «cambouis » dans lequel tout ethnographe plonge ses mains (mais aussi sa tête et son cœur...), Olivier Schwartz revient sur les modalités distinctes d'entrée sur ses terrains d'enquête : alors qu'il mène une enquête que l'on pourrait qualifier d' " incognito » (Dargère, 2012) dans le Nord, auprès d'hommes et de femmes qui sont ses voisin.e.s, il se présente comme un sociologue universitaire à la RATP. L'accès aux conducteurs et conductrices de bus de la région parisienne fait l'objet de nombreuses petites négociations en cascade, du haut vers le bas de la chaîne hiérarchique. Bien que ces deux enquêtes aient cours sur une temporalité longue, les pratiques de (non) négociation mises en œuvre par Olivier Schwartz diffèrent fortement et sont en partie liées aux rôles endossés sur chacun des terrains. Après avoir discuté des tenants et aboutissants « pratiques », mais aussi épistémologiques, de ses manières différenciées d'enquêter, il raconte très concrètement la façon dont il se présente et présente son travail, soulignant la part « d'inventivité » dont doit faire preuve l'ethnographe pour faire face aux imprévus ou aux lacunes inhérents à ce type de démarche. Il évoque la place centrale du statut de la parole dans ses enquêtes et aborde la question relativement peu débattue dans les arènes académiques des affects et des émotions dans la relation d'enquête, des raisons de ses insatisfactions, mais aussi de ses tourments et sentiments de culpabilité. Ces affects, loin d'être des parasites à la démarche d'enquête, sont au contraire de précieux « baromètres » pour résoudre certains dilemmes éthiques qui surviennent, notamment, au moment de négocier la sortie du terrain et de restituer les résultats de l'enquête. Il insiste sur l'importance de débattre collectivement de ces questions et affirme que sans « l'authentique souci des enquêté-e-s », la démarche ethnographique ne fait aucun sens.

\section{Les mains dans le cambouis ou la double impureté de la pratique ethnographique}

I : Comme nous vous l'avions annoncé, cet entretien vise à discuter de vos pratiques de négociation, qui sont au cœur de ce dossier de la revue Cambouis.

os : J'ai un peu hésité, comme je vous l'avais dit, à vous dire oui pour cet entretien, tout en étant très sensible à votre offre! D'abord parce que je suis un peu défensif - on me dit souvent, dans mon entourage, que je le suis 
trop! Mais il y a aussi le fait que je ne suis pas sûr d'avoir une très grande expérience sur cette question de la négociation. D'autres collègues l'ont certainement beaucoup plus que moi. Mais bon, on verra...

I : Ne vous inquiétez pas, nous sommes sûres qu'il y a matière à discussion. [Rires] Avant d'entrer dans vos pratiques d'enquête, nous aimerions toutefois revenir sur le titre de la revue Cambouis, la revue des sociologues aux mains sales pour avoir votre avis sur cette image de mécanos ou de cuisine...

os : Je me retrouve tout à fait dans ce titre. Bien sûr, je ne sais pas si ce que j'y projette correspond exactement à ce que la revue met dans ce terme, mais l'expérience que j'ai de la pratique ethnographique, c'est celle d'un travail très impur, d'abord épistémologiquement. Les sociologues, en tout cas c'est valable pour moi, qui font de l'ethnographie, qui pratiquent le « terrain », ont une ambition de produire du savoir. Je ne prononcerais peut-être pas le mot « science », parce que je n'y crois pas trop, mais en tout cas du savoir, de la connaissance. Mais en même temps, étant donné la situation dans laquelle on est quand on fait de l'ethnographie, il y a fréquemment un écart entre ce que l'on vise quand on a un tel objectif, et la situation dans laquelle on est, qui rend souvent difficile d'y parvenir. Je me retrouve donc dans cette idée de cambouis et bien que je n'en sois pas certain, je pense que c'est une image qui serait assez largement partagée par les collègues qui pratiquent ce type d'enquête. Quoique je ne sois pas dans les mêmes orientations théoriques que lui, le très bel article de Michael Burawoy (1998) sur « l'Étude de cas élargie » comporte sur ce point des développements qui me semblent très convaincants. Je précise que je n'adhère nullement (Burawoy de toute évidence pas non plus, d'ailleurs) à une version esthétisante ou littéraire de ce type de démarche. Le but n'est pas simplement de produire un beau récit. L'objectif est de produire de la connaissance, avec les formes de discipline intellectuelle, de rigueur, de précision que cela implique. Mais il me semble que, même si on a cette ambition haute, on est en même temps dans une situation qui rend souvent difficile de la réaliser. I: L'impureté dont vous parlez se situe au niveau épistémologique, mais l'ethnographie peut-elle être moralement ou éthiquement impure?

os : Il y a les deux. En fait je réagissais au terme « cambouis » tel que je supposais que la revue l'employait. Mais il peut aussi y avoir des situations d'impureté morale. Des collègues m'ont parfois... je ne dirais pas reproché, parce que c'est des objections vraiment faites très gentiment... mais ils m'ont parfois reproché d'en faire trop là-dessus, d'accorder trop d'importance à ces questions-là. Les quelques pages que j'avais écrites dans Le monde privé, il y a maintenant trente ans, sur le cynisme, m'ont valu des remarques de collègues : « Tu exagères, ce n'est pas si grave que ça, ce n'est pas la peine d'en faire un problème, ça peut se sociologiser ». Il me semble pourtant que ces questions se posent effectivement. Mais peut-être en reparlerons-nous... 


\section{Une inscription progressive dans le monde ouvrier}

I: Revenons sur vos expériences de négociation dans le monde privé des ouvriers, pour commencer. Est-ce que vous pourriez nous raconter comment vous avez procédé ? Quels sont les différents canaux par lesquels vous avez pu entrer en contact?

os :Je vais essayer. Comme le livre date de 1990, et qu'il est issu d'une enquête qui a été menée entre 1980 et 1985, beaucoup de temps s'est écoulé depuis. Il y a des choses dont je n'ai plus un souvenir très net, je ne sais plus toujours très bien comment elles se sont passées. Mais je vais tenter...

I: C'était il y a longtemps et cela s'est passé sur un temps long. Vous racontez que vous aviez l'envie de réaliser cette enquête et de faire de la sociologie sur ce sujet, mais que vous avez mis une année de négociation avec vous-même, avant d'aller frapper aux portes de vos voisins de HLM, avant de commencer cette enquête. Et puis vous vous êtes lancé et vous avez multiplié les points d'entrée : vous avez été introduit par une amie, vous êtes passé par les cafés, vous êtes passé par des associations syndicales notamment, le porte-à-porte, le voisinage...

os : C'est ça, vous maidez à démarrer. Disons que l'objectif que j’avais, même si on n'a jamais au départ des objectifs très clairs sur ce qu'on cherche, disons l'intention que j'avais, c'était d'essayer de travailler sur la famille, sur la sphère familiale, dans le monde ouvrier. Le sentiment que j'avais en effet était que - ce que je vais dire maintenant est vraiment daté, c'était la situation du tout début des années quatre-vingt -, s'agissant de la classe ouvrière et pour ce qui concerne la France, on avait acquis au cours des années 1960-1970 beaucoup de connaissances chez les sociologues sur la sphère du travail, sur l'industrie, l'usine, le travail ouvrier... Alors qu'on savait trop peu de choses, disons, par comparaison, sur la sphère familiale, la sphère du hors-travail, la « sphère privée » entre guillemets. Donc je voulais essayer de travailler là-dessus. Les choses ont bien changé depuis mais, à ce moment-là, c'était la situation.

Vous me demandez comment les choses se sont passées, comment l'idée de cette enquête est venue et quels canaux m'ont permis de m'insérer sur mon « terrain ». D'abord et avant tout, quand j'ai souhaité démarrer ce travail, j'étais enseignant, prof de philo dans l'enseignement secondaire, en lycée, à Hénin-Beaumont - la ville, aujourd'hui, de Marine Le Pen... mais à l'époque, tous les députés et les élus de la région étaient de gauche, souvent communistes. Donc j'étais prof de philo en lycée. C'était en 19791980, je le suis resté d'ailleurs jusqu'en 1990. Et là, j'avais une collègue, à quij'ai dédié ce livre, qui s'appelle Francine Kurzawski, qui était prof de lettres dans le même lycée, et avec qui je parlais beaucoup du souhait que j'avais de faire un travail de ce type. On se connaissait bien parce qu'on était collègues tous les deux, parce qu'elle était membre du Parti communiste français (PCF), comme moi. Je ne parlais, au lycée, à personne d'autre de ce souhait, parce que c'était un projet de faire autre chose que ce qui me reliait à mes collègues. C'était, d'une certaine manière, un projet de sortie du métier que j'exerçais à ce moment, mais pas au sens où je voulais aller plus haut, mais au sens où je voulais passer de la philosophie à la sociologie. Avec Francine Kurzawski en revanche, j'en parlais. Elle venait, elle, vraiment du milieu ouvrier du Nord, elle est fille de mineur. Elle habitait à ce moment dans un grand ensemble à côté duquel il y avait une zone pavillonnaire, un grand ensemble populaire, habité par une population ouvrière pauvre. C'est cet ensemble, ainsi que la zone pavillonnaire, qui est devenu le terrain de l'enquête. Elle y 
habitait depuis plusieurs années, elle y avait de nombreux liens, y était connue de nombreux habitants. La cellule du Parti communiste y était fortement implantée, et par ailleurs elle était en train de monter une association de défense des locataires. Francine m'a dit : «Écoute, si tu veux venir, je peux t'ouvrir des portes ». C'est très simple : ni l'enquête, ni le livre n'auraient été possibles sans elle. Les petites techniques que j'ai mises au point après, pour m'ouvrir d'autres portes, en particulier le porte-à-porte avec une espèce de questionnaire qui n'avait absolument aucune validité, ces petites techniques-là m’ont permis de développer mes contacts ; mais c'est Francine qui m’a donné les premières entrées, celles qui m’ont donné confiance dans le fait que c'était possible de mener cette enquête. Et par ailleurs ce n'est pas seulement les entrées qu'elle m'a données, c'est aussi un statut. C'est-à-dire que j'étais « le copain à Francine », si vous voulez... Et donc j'ai très vite eu une « case ». En fait, j'avais deux cases : j'étais « le copain à Francine », et j'étais « Olivier », qui est prof dans un lycée, qui a une vieille 2 chevaux (2 CV), qui habite là. Donc je suis venu résider là-bas pendant cinq ans tout en étant prof au lycée, lycée qui était à une dizaine de kilomètres du grand ensemble où j'habitais. Bien sûr, à ce moment-là, un prof c'était, pour la population ouvrière du grand ensemble, quelqu'un de beaucoup plus haut socialement. Ce n'était pas le Président de la République, mais c'était quand même quelqu'un de plus haut. Mais il se trouve, je ne l'avais pas fait exprès, que j'avais à l'époque comme voiture une vieille $2 \mathrm{CV}$. Je ne jouais pas les pauvres, c'est celle que j'avais depuis quelques années, donc j'avais cette $2 \mathrm{CV}$, que des gens finissaient par connaître parce qu'elle n'était vraiment pas belle... Donc si vous voulez, pour revenir à votre question, c'est ce statut, cette case, faits de plusieurs choses, c'est ce rôle, cette position qui ont certainement été l'élément le plus déterminant dans mon insertion sur mon «terrain », qui m'ont petit à petit ouvert des portes. Alors quand je dis « ouvrir des portes », ce n'est pas forcément la bonne expression, parce que les enquêtés je les voyais effectivement en allant frapper à leur porte et leur rendre visite, et leur dire bonjour, mais je les rencontrais aussi au café, situé dans la zone commerciale qui se trouvait tout près du grand ensemble. Tout cela m'a permis d'entrer en contact, de nouer des relations, et à partir de là, de parler, de passer du temps avec des gens...

\section{Absence de négociation et prises de rôles multiples : « J'étais le prof qui a la dedeuch, le copain de Francine, le voisin, le membre du PCF »}

I: Vous n’aviez donc pas annoncé aux personnes rencontrées que vous faisiez une recherche ? En fait, on pourrait dire que, dans cette enquête, vous n'avez pas eu à «négocier votre entrée »?

os : Oui, c'est parfaitement exact. Si on prend « négociation » au sens un peu strict du terme - même si on sait bien qu'il y a des dégradés - il n'y a eu quasiment aucune négociation dans cette enquête. Je n'ai eu à ce moment aucune expérience d'une vraie négociation, au sens fort. Aujourd'hui, dans mon travail à la RATP, je suis contraint, en permanence, de négocier. Mais pour tout le travail sur le Nord, qui a abouti au Monde privé des ouvriers, c'est une ethnographie sans négociation. Qui est entièrement passée par une inscription progressive dans des rôles, qui n'étaient pas d'abord des rôles de sociologue. Parce qu'après tout, l'inscription... la 
durée est un élément important, mais si elle se fait sous un rôle, sous un statut de sociologue, même s'il y a durée, il faut bien avoir négocié, puisqu'on est extérieur. Mais là, je n'étais pas, pour la majorité de mes enquêtés, dans un statut de sociologue, ni de chercheur, ça ne s'est pas passé comme ça - ça n'aurait jamais marché comme ça d'ailleurs, ça n'aurait rien dit aux gens...

I: Mais certaines personnes savaient quand même que vous écriviez quelque chose?

os : Certaines personnes, oui, savaient. Les quatre personnes des derniers chapitres par exemple, ainsi qu'un petit nombre d'autres. Mais la grande majorité de mes enquêtés ne savait pas ce que je faisais. J'étais avec eux dans des rôles, des statuts, qui n'étaient ni de chercheur, ni d'intellectuel, enfin au sens un peu officiel du terme, ni de sociologue. J'étais le copain de Francine, le prof qui a la dedeuch', le voisin, le membre du PCF. Et donc du coup il n'y avait pas négociation, puisque je n'étais le plus souvent pas perçu comme quelqu'un d'extérieur, qui veut mener une enquête. Et mon objectif était, en passant par le détour de conversations qui ne visaient pas explicitement les questions relatives à la famille, et qui au départ débutaient par des sujets beaucoup plus banals : « Tiens, salut, comment ça va ? Il fait beau aujourd'hui... », l'objectif était, petit à petit, au bout d'un quart d'heure, vingt minutes, une demi-heure, d'en savoir plus : «En fait tu m'avais dit qu'avec ton mari, ce n'était pas trop bien. Est-ce qu'il s'est un petit peu calmé ? ». Je n'ai d'ailleurs quasiment pas fait d'entretiens enregistrés. Mis à part un petit nombre d'entretiens, le livre est pour l'essentiel fabriqué à partir de petits bouts de conversations. Je revenais ensuite chez moi, et je prenais des notes. D'où le fait d'ailleurs que le sentiment d'instrumentalisation des enquêtés, je l'ai eu très vite et très fortement.

Mais pour revenir à la question que vous me posiez au départ, l'entrée majeure, elle m'a été donnée par Francine Kurzawski. Ça n'aurait pas pu être possible sans elle. Et puis, petit à petit, j'ai essayé d'élargir en m’appuyant sur d'autres types de rôles ou d'autres types de canaux. Il y avait l'idée de faire un petit peu de porte-à-porte avec un petit questionnaire de dix ou quinze questions, qui n'étaient que des prétextes. Et ça m’a permis de vérifier certaines informations... Par exemple, j'ai très vite été frappé, au cours des premières relations que j'ai eues grâce à Francine Kurzawski, par l'importance du personnage de la mère. Je ne cherchais pas la quantification, mais je voulais m'assurer que ce n'était pas quelques cas seulement. Donc dans le petit questionnaire, si les gens acceptaient d'y répondre (je ne sais plus quel était le prétexte que j'utilisais, quelque chose comme : «Voilà je suis étudiant, est-ce que vous auriez quelques minutes pour un questionnaire ?... », il faut dire qu'à l'époque je n'avais pas les cheveux blancs... « C'est pour un petit travail que je fais sur la vie quotidienne. »), et donc dedans, il y avait une question : « Est-ce que vous voyez souvent des personnes de votre famille? ». C'est là que j'ai vite constaté que : «Ah oui, ma mère, tous les jours. » Et donc je suis passé par ce petit questionnaire. Un autre statut par lequel je suis passé, c'est le statut de voisin. À ce sujet, une objection qui m'a souvent été faite après, et je pense qu'elle est légitime, c'est que j'étais tellement polarisé sur la question de la famille que j'ai très peu parlé de la sociabilité de HLM. Parce que ce n'était pas ça qui m'intéressait. Or en milieu populaire - mais je ne vous l'apprends pas, on peut se référer à Hoggart (1970) - et notamment en HLM, les gens sont aussi sur leur palier. Je croisais donc des voisins ou des voisines sur le palier, je les croisais en bas en attendant l'ascenseur, qui bien sûr une fois sur deux ne marchait pas... Donc on parlait. Et comme je l'ai mentionné, 
j'étais membre du Parti communiste à l'époque, ce qui m'a ouvert des contacts aussi. Les quatre personnages du dernier chapitre étaient tous membres du Parti communiste, et s'ils ont accepté le dialogue avec moi, souvent très intime, c'est d'abord et avant tout parce que Francine les connaissait intimement et qu'elle m'a présenté à eux, mais c'est aussi parce qu'ils étaient membres du PCF tous les quatre.

I: Reste la question de la négociation avec vous-même, vous racontez que vous avez mis une année avant de vous décider à débuter cette enquête?

os : Effectivement, entre le moment où Francine m'a dit: «Si tu veux voir, viens » et où je l'ai fait, il y a eu un an. J'habitais à Lille avec ma compagne dans un quartier relativement bourgeois, un quartier d'enseignants, de profs, de classes moyennes supérieures pourrait-on dire, et j'y avais un bienêtre, du confort. Et je craignais le HLM, parce que je viens vraiment de la bourgeoisie et je n'avais absolument pas l'habitude du HLM. D'autant plus que, si vous voulez, le HLM était constitué de deux grandes barres, et l'espace entre les deux barres était de quelques mètres, de sorte que tout mon appartement, par exemple, tout mon appartement donnait en face de l'autre barre. Et il n'y avait aucun rideau, pas de stores, rien. On était complètement exposés au regard des autres. De cela, je n'avais pas l'habitude...

I: Vous pouviez, quelque part, aussi observer... ?

os : Je pouvais, j'aurais pu, mais je n'osais pas, je ne l'ai pas fait. J'avais trop regardé Hitchcock! [Rires]... Donc j'ai surtout pensé à me protéger avec des rideaux... [Rires]

I : Mais vous avez dû convaincre votre compagne aussi ? Vous avez négocié avec votre compagne?

os : Alors oui. C'est exact. Mais en fait on était en train de se séparer, même si on ne le savait pas encore complètement. En fait, on séloignait progressivement l'un de l'autre. Donc de ce point de vue, la négociation a été plus avec moi-même.

\section{Négociations en cascade à la RATP : un enquêteur « fragile »}

I : Vous avez dit à plusieurs reprises qu'à la RATP votre pratique d'enquête est très différente et que, dans ce contexte, vous êtes tout le temps en train de négocier.

os : Oui. Dans cette enquête qui a démarré il y a plus de vingt ans, que j'ai plusieurs fois arrêtée puis reprise, oui, là, la situation est très différente, je peux dire que j'ai été et je suis sans arrêt en train de négocier... Ce sont le plus souvent de petites négociations, il n'y a pas eu de grande négociation d'entrée, mais il y a eu et il y a encore une série de petites négociations. La situation est très différente de celle que j'ai connue dans le Nord. Il y a à cela plusieurs raisons. La première, c'est que mon statut est explicitement cette fois un statut de sociologue extérieur à l'entreprise, et que ce n'est pas la RATP qui est venue me solliciter pour faire cette enquête, je la fais à ma propre demande, c'est moi qui suis demandeur. Il a donc fallu d'abord que j'obtienne l'autorisation de ce que l'on appelle à la RATP le « Département Bus » (la RATP est divisée en «Départements » correspondant aux différentes dimensions de son activité ; il y a deux Départements s'occupant du métro, un Département s'occupant du RER, un Département s'occupant des bus et du tramway, etc.). Il a donc fallu que j'aie un contact avec la direction du « Département Bus », pour me présenter, demander si l'on m'autorisait à faire ce 
travail. Et il m'a fallu du temps pour l'obtenir ; ça a été particulièrement le cas lorsque, il y a trois ans, la retraite m'en donnant la possibilité, j'ai décidé de me réengager fortement dans l'enquête, que j'avais arrêtée depuis trois ou quatre ans, mes charges d'universitaire ne m'en laissant plus le temps. Il a donc fallu que je réobtienne l'accord du Département, dont le directeur avait changé. Et ça a été très long. Non pas qu'il y ait eu refus, ou méfiance à mon égard, je ne le crois pas. Entreprise publique, la RATP n'est pas fermée aux sociologues, même si c'est une entreprise qui, en ce moment, change beaucoup, qui prend très fortement le virage des logiques entrepreneuriales, surtout au « Département Bus », qui va dans quelques années devoir affronter une concurrence générale avec le privé. Je n'ai pas été confronté à des refus au sens fort du terme. Mais il m'a fallu beaucoup de temps et d'insistance tout simplement pour obtenir une réponse à mes demandes, qu'on prenne contact avec moi.

I : L'autorisation auprès de la direction, vous l'avez obtenue par un entretien ou par un courrier?

os : C'est un entretien. Le courrier, j'ai essayé : aucune réponse. On ne me répondait pas.

I : Mais vous avez quand même obtenu un entretien.

os : Ça a été long ! J'ai pu finalement, grâce à l'aide qu'a eu la gentillesse de m'apporter un ancien responsable du Département, que j'avais rencontré une quinzaine d'années plus tôt, et qui occupe aujourd'hui des responsabilités élevées à la direction de la RATP, obtenir un entretien avec un responsable du « Département Bus », de la direction donc du Département, qui m'a reçu, et qui m’a donné son accord, ça a été capital. Cet accord m'a en effet permis de passer à une seconde étape. Le « Département Bus » s'occupe de la gestion des lignes de bus de la RATP sur l'ensemble du territoire que l'entreprise a en charge, c'est-à-dire Paris, la proche et la moyenne banlieue. La structure de base du Département est constituée par des dépôts d'autobus, répartis sur l'ensemble de ce territoire. Chaque dépôt (le terme officiel à la RATP est celui de « Centre Bus », mais c'est « dépôt » qui est le terme le plus ancien et le plus employé par les conducteurs) regroupe plusieurs centaines de conducteurs (la RATP emploie au total environ 15000 conducteurs) et gère les lignes de son secteur. Il y a au total une vingtaine de dépôts de bus gérant les lignes de Paris et de la banlieue. Ces dépôts sont placés sous l'autorité d'un directeur et d'une équipe de cadres. Le responsable du Département qui m'a reçu m'a donné l'autorisation de prendre contact avec des directeurs de dépôt, en me recommandant de son accord : «Je vous écris avec l'accord de... ». Cet accord a donc été décisif. Mais il n'était en même temps que le début d'un long processus. J'ai contacté de nombreux directeurs et directrices de dépôts, mais avec, à nouveau ici, des résultats inégaux. Plusieurs m’ont répondu, m’ont reçu, m’ont autorisé à venir travailler sur le dépôt qu'ils dirigeaient, certains m'aidant même immédiatement à obtenir certains contacts ; ma dette à leur égard est grande, c'est leur accueil qui a rendu mon enquête possible. D'autres n'ont pas répondu, ou seulement à la suite de nombreuses sollicitations de ma part. J'ai dû beaucoup, et je continue à beaucoup écrire, solliciter, réécrire, patienter... Les lignes et les dépôts de bus de la RATP desservent en effet des secteurs extrêmement différents sociologiquement de l'Ile-de-France. Un dépôt situé en Seine-Saint-Denis, le département le plus pauvre de l'Ile-de-France, et un autre gérant des lignes du centre de Paris, ce n'est, à bien des égards, pas du tout la même chose sur le plan des situations de travail pour les conducteurs. Il était donc important pour moi, sans viser la représentativité, d'obtenir l'accord de directeurs de dépôts reflétant suffisamment la diversité des 
contextes. C'est là que les choses ont été à divers moments difficiles, mes démarches, fructueuses pour obtenir des contacts dans les dépôts de tel ou tel secteur, n'aboutissant à rien dans d'autres, situés dans des contextes qu'il me semblait pourtant important d'étudier. Mais encore une fois, ce n'est pas la RATP qui est venue me chercher pour effectuer cette enquête, c'est moi qui suis demandeur...

Il y a par ailleurs d'autres raisons qui expliquent que j'aie été et sois continument en négociations. Il y a d'abord le fait que les cadres des dépôts changent. J'ai plusieurs fois été confronté à des situations où le directeur ou la directrice qui m'avait donné son accord sur son dépôt part, change d'affectation, donc il ou elle est remplacée par quelqu'un avec qui il faut reprendre contact, et qui ne me répond pas. Ensuite il y a le fait que les négociations se déroulent à plusieurs niveaux. Une fois que j’ai obtenu l'accord du directeur ou de la directrice du dépôt, ce qui m’intéresse, c'est de pouvoir enquêter sur des lignes, de parler avec les conducteurs des différentes lignes. Si le directeur ou la directrice sont d'accord, les choses se passent souvent comme suit. Chaque ligne est placée sous la responsabilité d'un agent de maîtrise qu'on appelle le chef de ligne, ou encore le REL, le ou la « responsable de l'équipe de ligne ». Ce que le directeur ou la directrice de dépôt me disent, s'ils sont d'accord pour que j'enquête, c'est : «Je n'ai pas d'opposition », «Vous pouvez prendre contact de ma part », «Je vous donne le nom et les coordonnées des responsables de telle et telle ligne, vous pouvez prendre contact avec eux en leur disant que je suis d'accord. Mais ce sont eux qui voient, sur leur ligne. ». À nouveau ici, c'est l'inconnu. Je prends contact avec le chef de ligne, qui me répond ou qui ne me répond pas, car il est souvent débordé. Une ligne de bus, c'est fréquemment une soixante de chauffeurs au moins ou davantage, qui sollicitent beaucoup leur responsable. Les chefs de ligne ont en charge une multiplicité de fonctions sur leur ligne, ce qui pèse sur eux est aujourd'hui très lourd, ils n'ont pas le temps. C'est dans ces conditions qu'il faut que j'obtienne leur accord. À nouveau ici, comme pour les directeurs de dépôts, c'est grâce à l'accueil, à la compréhension, à la bienveillance de plusieurs chefs de ligne que j'ai pu mener ce travail. Mais là aussi il faut souvent écrire, patienter, réécrire. Et par ailleurs, là encore, il y a les cas où le chef de ligne change d'affectation au cours de l'enquête. C'est donc cela, si vous voulez, ma situation et c'est cela qui la rend fragile : une multiplicité de petites négociations, avec des interlocuteurs dont certains sans doute ne veulent pas de moi, mais qui surtout sont souvent tout simplement débordés, négociations dont je ne peux pas faire l'économie et dans lesquelles je suis en position de demandeur. En même temps, aucune de ces petites transactions (à l'exception de celle, initiale, avec le responsable du Département) n'est absolument décisive, aucune n'est absolue. Si l'une échoue, je peux tenter ma chance dans un autre dépôt, avec un autre directeur, avec un autre chef de ligne. L'échec n'entraine rien de définitif. Sauf que ma compagne pourrait vous dire que j'en suis parfois malade pendant huit jours... Parce que j'attends une réponse qui ne vient pas... [rires]

I : Et pourquoi avez-vous besoin de l'accord du directeur de dépôt ou du chef de ligne? Est-ce que vous ne pourriez pas simplement aller dans les bus si ce sont les chauffeurs qui vous intéressent?

os :Très bonne question. Oui, pardonnez-moi, effectivementj'oublie d'expliquer les préalables. Si vous voulez, ce que je peux très bien faire dans les bus, c'est y aller de façon anonyme, comme n'importe quel usager, et observer ce qui se passe... Je l'ai fait bien sûr, et je le fais. Mais ce qui m'intéresse par ailleurs, c'est de pouvoir m'entretenir avec les conducteurs. Or pour qu'ils acceptent que je les accompagne pendant leur travail et que je 
m'entretienne avec eux, il faut qu'ils soient assurés que, s'ils acceptent - sachant que lorsque je les aborde, je précise que rien ne les oblige à accepter de parler avec moi -, ils ont l'accord de leur chef de ligne pour répondre à mes questions.

\section{La négociation au volant : s'entretenir en situation de travail}

I: Donc l'enjeu pour vous est de faire l'entretien sur le temps de travail ?

os : Oui, exactement, je fais les entretiens avec les conducteurs dans le bus, pendant qu'ils sont au volant. En fait, ma pratique est la suivante : elle consiste à les aborder lorsqu'au début de leur service, ou après une pause, ils vont commencer un trajet, et à leur dire : « Voilà, je suis sociologue, je travaille pour l'Université, je fais une étude sur le métier de machiniste (c'est le terme par lequel on désigne à la RATP les conducteurs). Je m'adresse à vous avec l'autorisation du chef de ligne. Est-ce que vous accepteriez que je vous accompagne un bref moment pour parler avec vous du métier?». Donc effectivement, oui, s'ils acceptent - et c'est presque toujours le cas - l'entretien a lieu pendant leur travail. C'est bien sûr aussi pour cela que cela ne peut se faire qu'avec l'autorisation du chef de ligne. Je me place debout, près d'eux, avec à la main un petit dictaphone pour enregistrer s'ils acceptent - donc là aussi, il y a négociation -, et je leur propose de parler avec eux de leur travail et de leur vécu du travail. J'ai eu très vite recours à cette manière de faire, parce que si le responsable - ou la responsable, il y a aussi des femmes - de ligne m'y autorise, elle présente un intérêt considérable :je ne demande pas aux conducteurs de m'accorder du temps sur leur temps personnel. C'est un avantage très remarquable de ce métier pour l'enquêteur que de rendre possible une telle situation! Et comme c'est un travail qui, tout en étant fatigant - la conduite d'un bus en zone urbaine très dense, comme l'est la région parisienne, est fatigante -, est un peu routinier, beaucoup disent, quand l'entretien se termine, qu'ils ont été contents parce que ça a rendu leur trajet sur la ligne plus agréable, le temps est passé plus vite. Naturellement, vis-à-vis du chef de ligne qui me l'autorise, je prends des engagements, celui de ne pas perturber le travail du conducteur, et celui, lorsqu'il arrive à une station, de le laisser s'occuper des voyageurs qui montent dans le bus (la RATP accorde aujourd'hui une grande importance à cette question). À chaque station, je me mets donc un peu en retrait, et je laisse le conducteur se tourner vers les « clients ». Et je reprends après avec lui.

I: Et il y a-t-il des conducteurs qui sont mal à l'aise du fait qu'il y ait un public, des usagers, des « clients » dans le bus?

os : Non, parce que j'essaie de choisir des heures où il n'y a pas trop de monde dans le bus. Si jamais tout à coup le bus est bondé, même à l'avant, je me mets alors en retrait, j'attends que des gens descendent, que le bus, l'avant du bus en tout cas, se vide un peu, que je puisse retrouver un espace « privé » avec le conducteur, et je reviens le voir. Évidemment ça donne dans certains cas un entretien haché, avec des coupures, des interruptions, mais il faut faire avec... Et puis c'est une telle chance pour 
moi de pouvoir parler avec les chauffeurs, discuter avec eux, passer du temps avec eux, et cela en plus dans leur environnement « naturel », quand ils sont au volant, à leur travail...

I : Vos entretiens sont donc hachés, morcelés?

os : C'est morcelé, mais j'ai appris à faire avec. Et il faut voir aussi un autre intérêt, à mon avis important, de cette situation pour l'entretien. Les conducteurs sont des travailleurs subordonnés, généralement peu diplômés, dont certains peuvent craindre, pour reprendre leur expression, de «ne pas savoir parler ». La rencontre avec un interlocuteur qui leur apparait comme un intellectuel n'est pas forcément facile pour eux. Mais là, c'est moi qui vais sur leur terrain, et non eux qui viennent sur le mien. On n'est pas dans un bureau. Ils sont à leur travail. Ce sont eux qui sont aux commandes. C'est à moi de m'adapter à eux. Je pense que cette situation favorise en fait leur prise de parole, elle atténue ce que l'entretien, en face à face, pourrait avoir d'insécurisant pour certains d'entre eux. C'est pourquoi il est tellement précieux pour moi d'obtenir cet accord du chef de ligne. S'il m'autorise cette pratique de l'entretien à bord du bus, c'est une ressource décisive pour l'enquête qui m'est donnée. À charge pour moi, comme je l'ai dit, de prendre avec lui des engagements. Un autre engagement que je prends également est de ne jamais venir sur la ligne sans l'informer à l'avance, de façon à ce qu'il sache et qu'il puisse toujours me dire si, sur la ligne, le jour que j'envisage, pour quelque raison que ce soit, ne lui parait pas souhaitable.

I : On pourrait dire que le chef de ligne vous oriente quand même?

os : En fait, très sincèrement, non. Je prends cet engagement pour qu'il soit clair que mon interlocuteur conserve toujours la possibilité de me dire non. Mais aucun des chefs de ligne qui m'ont donné leur accord pour ces entretiens ne m'a demandé de renoncer à ma venue tel jour sur la ligne. Et surtout, jamais aucun d'entre eux ne m'a demandé de choisir lui-même les conducteurs que je rencontre. Le principe est que j'arrive sur la ligne, j'aborde un premier conducteur au moment où il commence son trajet, je l'accompagne s'il est d'accord pour un entretien à bord du bus, puis je fais de même avec un second, éventuellement ensuite un troisième si j'en ai la force. Plusieurs dizaines de conducteurs travaillant chaque jour sur une ligne, le chef de ligne ignore quels sont ceux que je rencontrerai, et encore une fois aucun n'a jamais cherché à m'imposer quoi que ce soit sur ce plan. Je dois beaucoup m'impliquer en amont, pour les raisons que j'ai dites, pour parvenir à entrer en contact avec le chef de ligne et pour obtenir son accord de principe, je n'obtiens pas toujours son accord (tel responsable de ligne qui me l'avait d'abord donné ne répond plus ensuite à mes mails lorsque je lui propose une première date, tel autre me le donne, puis cesse de me répondre au bout de quelques passages de ma part), mais ceux qui m'ont donné leur accord m'ont tous laissé une complète liberté dans l'accomplissement du travail et n'ont jamais cherché à en contrôler le déroulement ou les résultats. 


\section{Se présenter et présenter sa recherche en quelques secondes : la stratégie $\mathrm{du}$ « feu rouge »}

I : Vous avez expliqué que cette enquête se déroule sur un temps long : vous venez, vous repartez... Mais quand vous reprenez contact, est-ce que vous devez repasser par un bout de cette chaîne ou vous pouvez directement prendre contact avec le directeur de ligne et puis ça se fait peut-être facilement?

os : Non, si j’ai longtemps cessé de fréquenter un dépôt, en général le directeur ou la directrice a changé, il faut donc tout recommencer. Par exemple, j'ai commencé cette enquête il y a vingt ans, puis je l'ai interrompue, puis reprise, et je n'ai jamais pu retourner sur le dépôt sur lequel j'avais commencé. Il s'agissait d'un très gros dépôt de banlieue. J'ai commencé mon enquête sur ce dépôt, au milieu des années quatre-vingt-dix, je l'ai fréquenté de façon régulière pendant plusieurs années. Puis j’ai arrêté, certes non pas de façon brutale, mais progressivement, une raison majeure étant les difficultés croissantes que je rencontrais pour poursuivre ce travail étant donné les charges qui étaient les miennes comme universitaire (préparation des cours, suivi des étudiants, suivi des doctorants, jurys de thèses, etc.). En 2016, j'ai repris cette enquête, intensivement. J'ai alors souhaité, tout en prenant contact avec d'autres dépôts, reprendre contact avec celui-ci, où j'étais parvenu à nouer de nombreux liens avec des conducteurs. Je n'y suis jamais parvenu. J'ai écrit au directeur, patienté, puis réécrit à deux reprises, tout cela par mail, je n'ai pas eu de réponse. C'est en ce sens, comme je le disais plus haut, que je suis fragile. Le fait d'être dans cette situation récurrente de petites négociations me met dans une situation fragile. Rien n'est jamais acquis. Tout doit toujours être repris...

I: Du coup concrètement, il y a plusieurs niveaux : il y a le responsable du département, le directeur de dépôt, le chef de ligne, le conducteur de bus et après la négociation de l'entretien enregistré... Avec les conducteurs, comment faites-vous?

os: Oui, voilà, il y a tous ces niveaux. En ce qui concerne les conducteurs, disons qu'avec le temps, j'ai adopté une manière de me présenter à eux, qui dans l'ensemble, me semble-t-il, passe relativement bien auprès d'eux.

I : Et que dites-vous ? On aimerait savoir ! [Rires]

o.s. : Je fais ainsi. D'abord, quand le conducteur monte dans son bus (à la suite d'une pause, ou pour commencer son service), je le laisse s'installer, et démarrer le bus. Je ne dois pas lui « tomber dessus ». Après, une fois qu'il est parti, je ne m'adresse pas à lui tout de suite. J'attends un feu rouge, plutôt même un feu qui vient de passer au rouge, de façon à ce qu'il puisse prêter attention à ce que j'ai à lui demander. Puis : «Bonjour Monsieur, ou Madame, est-ce que je peux vous déranger quelques instants? » Parce qu'ils ne supportent pas les clients qui entrent dans le bus: «Vous partez à quelle heure? », sans aucun bonjour, sans aucune civilité. «Vous partez à quelle heure? », « Vous allez où ? ». Donc : « Bonjour Monsieur, est-ce que je peux vous déranger quelques instants? », «Je vous écoute ». Je me présente alors : «Je m'adresse à vous avec l'accord du Chef de ligne, Monsieur Untel, Madame Unetelle. Je ne suis pas de la Régie. Je suis sociologue, je travaille pour l'Université. Je fais depuis un moment, à ma demande, une étude sur le métier de machiniste. On m'a autorisé, pour la journée, à venir sur la ligne et à aborder les machinistes qui sont aujourd'hui sur la ligne, et à vous demander si je peux vous accompagner un petit moment... Est-ce que vous accepteriez? 
Je ne m'impose pas, vous n'êtes pas du tout obligé, c'est seulement si vous acceptez ». Voilà, c'est à peu près ça. Pas de terme technique, sauf « sociologue » (mais il faut bien que je me donne une identité, qui ne soit si possible ni journaliste ni « psy »), pas de formulation (tout au moins je l'espère) qui intimide (je ne suis pas « chercheur», je «travaille pour l'Université »). Dans $90 \%$ des cas, la réponse est positive. J'ai quelques refus, un tout petit nombre vraiment. Quelques anciens, un peu désagréables, qui sans doute, quoi que je fasse, me perçoivent comme un agent de la hiérarchie. Un qui, un jour, m'a regardé fixement dans les yeux, pendant plusieurs secondes, sans répondre ni dire quoi que ce soit. Je l'ai rencontré ensuite dans un autre contexte, dans le cadre de réunions syndicales de conducteurs auxquelles j'assiste par ailleurs, et à ce moment-là on s'est très bien entendus. Quelques refus aussi de jeunes, qui débutent dans le métier, me disant : «C'est pas que je ne veux pas, mais je débute, et le bus, les clients, les voitures, les piétons, les cyclistes, et tout, c'est vraiment compliqué... ». Mais sinon, l'immense majorité, je peux même dire la quasi-totalité, c'est oui. Les conducteurs ont l'habitude du contact, ils font un métier de service. Et par ailleurs, parler d'un métier dont ils pensent, assez justement, que les difficultés sont mal connues à l'extérieur, ne leur déplait pas.

I : Mais vous êtes équipé comment? Vous êtes debout quand même pendant relativement longtemps...

os : Oui, je suis debout près du conducteur, le plus souvent pendant au moins deux heures avec chacun. Je laisse passer l'heure de pointe, parce qu'à ce moment-là il y a souvent tellement de monde dans le bus que beaucoup de gens se retrouvent debout tout près du conducteur, ce qui ne crée pas des conditions pour parler avec lui. Je commence vers 9 h 30 et je vais jusqu'à $17 \mathrm{~h} 30$. Je fais des pauses régulièrement, je vais boire des cafés. Et j'ai avec moi ma sacoche, qui contient notamment un pain au sucre - ce qui, pour moi, est fondamental ! [rires] - et, bien évidemment, mon dictaphone.

I : Et le dictaphone... Vous le posez ? Vous le tenez?

os : Je le tiens à la main.

I : Et comment ça se passe pour l'enregistrement?

os: Alors, d'abord, si le chauffeur m'a donné son accord, je lui demande : « Estce que je peux déposer ça [ma sacoche] contre votre vitre? » [la vitre avant du bus]. « D'accord, pas de problème ». Et alors vient le moment...

I : De négocier l'enregistrement...

os : Voilà. « J'ai une faveur à vous demander. J'ai toujours avec moi un petit dictaphone. Est-ce que vous accepteriez que je le sorte, pour enregistrer, pour ne pas perdre ce que vous me direz? Je ne vous demande pas votre nom, et je n'ai aucune question à vous poser sur la hiérarchie, ni sur qui que ce soit. Il n'y en aura aucune. » Et ça, c'est vrai, je n'en pose aucune. Même si bien sûr, si mon interlocuteur aborde le sujet, et ce n'est pas rare, inutile de vous dire que je ne refuse pas... « C'est uniquement sur le vécu du métier, et sur ce que vous avez fait avant. Est-ce que vous accepteriez ?». Évidemment c'est un moment de tension intérieure pour moi. J'ai quelques secondes pour essayer de faire en sorte que ma demande soit acceptée...

$\mathrm{I}:$ Et ils acceptent?

os : Il ne m'est pas complètement facile de vous répondre, parce qu'il m’arrive en ce moment, depuis seulement quelques mois, je dirais deux mois, quelque chose de nouveau. J'ai commencé, je vous le disais, cette enquête il y a une vingtaine d'années. Durant toutes ces années, et jusqu'à il y a quelques mois encore une fois, j'ai fait d'innombrables entretiens, et je peux dire que ma demande d'enregistrement était presque toujours 
acceptée. Beaucoup me disaient tout de suite : «Oui, d'accord pas de problème ». D'autres hésitaient un peu : «Si vous voulez... mais qu'estce que vous allez en faire après? », ou « Je ne sais pas si je vais savoir parler... », mais je leur proposais qu'on « essaie », « et si jamais vous sentez que ça vous gêne, on arrête tout de suite », et presque toujours ils acceptaient.

Or, je constate que depuis deux mois environ, les refus sont nettement plus fréquents. Un grand nombre d'acceptations heureusement, mais beaucoup plus de refus, ce qui n'empêche pas l'entretien de se dérouler le plus souvent très bien, mon interlocuteur parlant volontiers, m'apportant beaucoup de choses (des entretiens qui ont commencé par un refus peuvent se terminer, trois heures après, dans un climat chaleureux entre mon interlocuteur et moi), mais déclinant au départ ma demande d'enregistrement (« Non, je préfère pas », «j'aime mieux pas », ou plus vivement : «Ah non ça je veux pas »). Je me demande s'il ne s'agit pas là d'une manifestation de la montée de cette méfiance à l'égard de tout ce qui peut sembler comporter un risque d'instrumentalisation de l'individu par d'autres (que ce soit par les politiques, par les médias... et donc aussi par moi...), dont on sait qu'elle est extrêmement vive aujourd'hui en France, comme on a pu le voir notamment lors du mouvement des Gilets jaunes. Je ne dispose pas néanmoins encore du recul, ni d'un nombre suffisant d'expériences de ce type, pour savoir exactement de quoi il s'agit.

Cela étant, pour en revenir à votre question et prendre les choses plus globalement, oui, l'immense majorité des conducteurs à qui je l'ai demandé au cours de toutes ces années ont jusqu'ici accepté ma demande de sortir le dictaphone, ce qui me permet de disposer aujourd'hui d'un grand nombre d'entretiens enregistrés.

\section{Les affects dans la négociation}

I: Pour mettre en place cette «stratégie de l'approche du conducteur au feu rouge » dont vous parliez, est-ce que vous avez eu des ratés avant, ou c'était prévu, d'emblée vous aviez pensé à ça ?

os: Alors, j'aurais du mal à vous dire comment ça s'est fait, je ne m'en souviens plus suffisamment. La mise au point s'est faite, je pense, progressivement. Ce que je peux vous dire en revanche clairement, c'est que ces situations de demande d'entretien à des inconnus ont toujours suscité chez moi un mélange de désir et d'angoisse. Je ne suis jamais tranquille quand je vais à un entretien, ou sur mes «terrains ». Je suis toujours anxieux. Peut-être un peu moins aujourd'hui qu'avant, mais l'inquiétude, la crainte suscitée par cette rencontre avec un inconnu et cet entretien que je vais devoir lui demander est toujours là. Crainte d'aborder le conducteur, qu'il me dise non, de me faire jeter, de me faire mal recevoir... Je vous parle de cela parce que, par rapport à votre question, je n'ai pas le souvenir d'échecs clairs et nets qui m'auraient conduit à changer mes manières de faire, mais j'ai le souvenir d'une angoisse, que je porte d'ailleurs toujours en moi, qui m’a incité à multiplier les précautions, 
les prudences, dans la manière d'entamer le premier contact, d'aborder mes interlocuteurs potentiels. Je crois que c'est beaucoup comme ça que les choses se sont passées.

I: Vous avez dit à un moment donné que, quand vous échouiez dans une négociation, vous mettiez plusieurs jours à vous en remettre...

os : Sans fuir votre question, j'aimerais d'abord la relier à une autre, c'està-dire à celle des affects. J'ai beau faire ce métier depuis longtemps, chacun des actes qu'il implique (rédaction d'un mail, demande d'un contact ou d'un entretien, et bien sûr quand je vais sur mes « terrains ») continue de me mobiliser fortement affectivement. Il y a, comme je le disais, l'angoisse que ça ne marche pas, que les personnes auxquelles je m'adresse ne m'acceptent pas, que la relation avec elles échoue, enfin voilà... Et pour revenir à votre question plus précisément, oui, quand je comprends qu'après trois ou quatre tentatives que j'ai faites pour obtenir un contact qui me semblait important (avoir accès à tel type de situation, de dépôt, de ligne ou de secteur...), je n'y parviendrai pas, mes demandes étant restées sans réponse, j'ai souvent du mal à supporter la situation, à supporter la déception (je n'ai pas pu obtenir ce quej'espérais malgré mes tentatives) et l'inquiétude (comment vais-je faire, puisque j'estimais ce type de contact important)... Il faut, bien sûr, l'accepter, faire avec... D'autant plus, comme je le disais, que c'est la contrepartie de ma situation à la RATP. Je ne suis pas missionné par la RATP, ce qui est d'abord une chance, car cela me donne une grande liberté. Mais la contrepartie est que, quand je frappe à une porte, je frappe à la porte de quelqu'un qui n'a absolument aucune obligation de me répondre. Il faut donc l'accepter, se dire aussi que je peux, à la place des entrées que je n'ai pas pu obtenir, essayer d'en trouver d'autres qui pourront jouer le même rôle, que je peux chercher à ouvrir d'autres portes. Je le fais bien sûr. Mais tout cela a pour conséquence que dans cette enquête que je mène depuis des années, il y aura eu, et il y a toujours, à la fois beaucoup de plaisir, de très nombreuses rencontres, affectivement très fortes pour moi, avec des conducteurs au moment des entretiens (ce sont ces rencontres qui font, pour moi, de l'enquête, une passion...) et en même temps du tourment...

I: Du tourment y compris quand on a trente ans, quarante ans de carrière ?

os: Ah oui, absolument. Je ne chercherai pas à atténuer la formulation. Tourment avant d'aller à un entretien, je l'ai dit, mais tourment aussi après, d'une autre nature, car il y a aussi les entretiens que j'ai le sentiment d'avoir mal gérés par ma faute. Ça me hante beaucoup aussi. Il y a quinze jours encore, en réécoutant un entretien avec un conducteur, en écoutant mes propres questions et en voyant la quantité de choses qu'il avait dites, sur lesquelles j'aurais dû le relancer et je ne l'ai pas fait... J'étais consterné. Le tourment c'est aussi cela, le sentiment désolé d'avoir laissé passer des occasions... Je l'écris d'ailleurs dans la marge quand je retranscris : «j'ai été mauvais, j'aurais dû, là... je ne lui ai pas posé cette question-là. » Alors ça, c'est récurrent.

I : Oui... Pour revenir à ce que vous mentionniez avant, quelque part c'est comme si les sociologues de terrain devaient aussi être capables de rebondir, et de faire preuve d'innovation, de créativité, d'originalité, dans les pistes qu'ils explorent ou qu'ils envisagent... et c'est quelque chose qui est difficile à transmettre...

os : Je suis tout à fait d'accord avec vous. On n'arrête en fait pas de bricoler, de chercher des entrées substitutives... Il me semble qu'on fait tous et toutes cela. C'est-à-dire, on essaie en fait de combler les manques d'un terrain qui est toujours profondément lacunaire, parce que le propre de ce type d'enquêtes, c'est qu'on est dépendant des réponses, 
des acceptations des enquêtés, et donc on essaie de combler les failles ou les manques d'une source essentiellement lacunaire en cherchant des solutions détournées pour avoir accès à un équivalent. Oui, je suis vraiment d'accord !

\section{Négocier, enquêter : des pratiques socialement situées}

I : Pour revenir une fois encore à vos démarches, auprès de responsables de la RATP, pour entrer en contact et formuler une demande, comment faites-vous ? Pourriez-vous en dire un peu plus?

os : Je passe par le mail. Des mails longs, en général. J'écris facilement à mes interlocuteurs des mails de vingt-cinq à trente lignes. Je n'ai jamais su faire court! Je me présente, moi-même ainsi que ma démarche. J'explique ce que je fais, et ce que je sollicite, auprès de mon interlocuteur, la possibilité de faire à l'intérieur de la structure sur laquelle il a autorité. Je suis long aussi parce que, tout en présentant ma démarche, je cherche à introduire des atténuateurs de ce qui peut inquiéter dans ma demande. J'euphémise. Mon milieu bourgeois d'origine m’a appris à le faire ! Si j'écris par exemple à un responsable de ligne, pour lui demander si je peux venir sur sa ligne, aborder des conducteurs, faire des entretiens avec eux, je ne dis pas « entretien », je dis « parler avec, un peu », je dis comment je fais sur d'autres lignes, dans d'autres dépôts, je précise que je veillerais constamment, si cette autorisation m'était donnée, à ne perturber en rien le travail des conducteurs (tant sur le plan de la conduite du bus que sur celui de l'attention qu'ils doivent aux « clients »), que l'entretien porterait uniquement sur le vécu du métier, qu'il n'y serait pas question de la hiérarchie, ni de politique, etc. Ces précautions, ces atténuateurs dans mes explications contribuent à me rendre long dans mes mails... Mais je les crois importants, pour rassurer mes interlocuteurs quant au fait que je ne serai pas un facteur de perturbation, même s'il existe certainement d'autres manières de faire...

I : Toutes ces précautions, ces formules de politesses que vous utilisez à l'oral, comme à l'écrit, vous les avez rattachées tout à l'heure à votre milieu social, vous parliez de votre aptitude à l'euphémisation. Qu'en a-t-il été des effets de votre milieu social, un milieu bourgeois d'origine comme vous le disiez, dans vos enquêtes ? Vous a-t-il trahi dans certains cas, vous qui enquêtez dans des milieux populaires? Ou est-il aussi arrivé qu'il vous aide?

os : Les deux ! En tout cas je pense. Que mes enquêtés l'aient perçu à certains moments et qu'ils en aient retiré le sentiment d'une nette distance entre eux et moi, ça ne fait à mon avis guère de doute. Je peux vous raconter une anecdote. Il y a une expression qu'on emploie souvent dans mon milieu, c'est « est-ce que je peux vous demander... », c'est-à-dire qu'au lieu de demander quelque chose à quelqu'un : « Où passez-vous vos vacances? », on commence par demander si on peut demander, « est-ce que je peux vous demander... ». De l'euphémisation à l'état pur, donc! C'est une expression de famille, de mon milieu, c'est plus fort que moi, je ne fais pas attention, c'est social... Et donc je me rappelle, un jour un conducteur que j'interviewais me disait : «Écoutez, ne me demandez pas si vous pouvez me demander, demandez-moi ! Ce jour-là, j’ai compris comment une certaine forme de politesse excessive chez moi, je veux dire avec trop de circonvolutions dans les demandes, d'abord trahissait vraisemblablement mes origines, et pouvait agacer certains 
de mes interlocuteurs! Et en même temps, mon milieu m’a aussi aidé. Je ne dis pas cela pour le défendre, mais c'est une manière de reconnaître mes dettes. Je viens de la bourgeoisie, mais pas de n'importe quelle fraction : une bourgeoisie d'État, de la haute fonction publique, une bourgeoisie juive et humaniste aussi... Mon père a été, pendant toute sa vie professionnelle, très directement ancré, par ses fonctions, ses passions, ses intérêts, dans les questions concernant la formation permanente auprès des personnes peu diplômées. Il a passé toute une partie de sa carrière à s'intéresser à la formation permanente des ouvriers de la région minière et sidérurgique de l'Est de la France. J'ai grandi là-dedans. J'ai reçu de mon père un intérêt extrêmement fort pour ce que l'on appelait autrefois - le terme est certes très daté - la « question sociale », ainsi que la conviction que, même quand on venait de la bourgeoisie, la rencontre avec les ouvriers était possible. C'est quelque chose que j'ai raconté dans un entretien avec Nicolas Renahy (2006). Donc je pense qu'il y a les deux, mon milieu m'a à la fois éloigné, trahi à certains moments, et aidé, fortement aidé même, à d'autres, dans mes rapports avec mes enquêtés.

I : Votre milieu social, mais aussi par exemple votre sexe, notamment dans l'enquête sur le monde privé des ouvriers - vous parlez du rapport des femmes à leur mère -, ça ne vous a jamais empêché d'accéder à certaines informations, avec certaines voisines?

os : Ça aurait certainement pu ! Mais la chance, énorme, que j’avais était que je n'étais pas seul sur mon «terrain ». J'échangeais régulièrement avec Francine Kurzawski, qui m'en avait ouvert les portes, qui m'avait donné les premiers contacts, qui résidait, elle aussi - depuis bien plus longtemps que moi, puisque c'est elle, comme je l'ai dit, qui m’avait incité à y venir - dans le grand ensemble où j'enquêtais. Et elle percevait de nombreuses choses que je ne percevais pas. C'est elle qui m'a alerté sur de multiples aspects du monde des femmes, auxquels je n'aurais jamais été attentif sans elle. En ce sens, oui, pour répondre à votre question, il y a bien un sexe de l'enquêteur, l'enquêteur que j'étais était « genré », et il serait, très certainement, passé à côté de multiples choses s'il n'avait pas bénéficié du regard de cette observatrice hors pair qu'était Francine Kurzawski.

I : Justement, par rapport à cela, comment s'y prend-on pour « observer » la sphère de l'intime, du privé, dans votre cas, lorsqu'on est un homme?

os :Je ne me sens pas en mesure de répondre à votre question sur sa dimension sexuée, c'est-à-dire : «lorsque l'on est un homme ». Et je le regrette, parce que c'est une belle question! Mais je dois confesser ne pas y avoir suffisamment réfléchi. Je vais donc prendre votre question sur un plan plus classiquement (et étroitement) méthodologique. Vous me demandez comment j'ai essayé « d'observer l'intime ». En fait, je ne dirais pas que j'ai cherché à l'observer. Je crois que j'ai d'abord et avant tout essayé de parler. Parler avec mes enquêtés pour comprendre, pour tenter d'accéder, à partir de ce qu'ils me disaient, au sens que les différents éléments constituant leur univers intime avaient pour eux. Par exemple, à propos de la relation des femmes avec leur mère : pourquoi ? Pourquoi ce besoin de leur mère en dehors de leur mari ? Qu'est-ce qu'elles ne trouvaient pas du côté de leur mari que peut-être elles trouvaient du côté de leur mère ? Je cherchais d'abord à parler, pour tenter d'accéder à du sens. La conséquence est que mon livre comporte peu de descriptions précises de ce que ces femmes faisaient avec leur mère, de ce dont elles parlaient, de la manière dont ces moments passés avec elle s'écoulaient... Est-ce que c'est ainsi que je ferais si j'entreprenais aujourd'hui ce travail ? À la fois oui, et non. Oui, au sens où il y a une idée de l'ethnographie qui a toujours été la mienne, et qui le demeure, même si je ne la formulerais 
plus de la même manière. Pour moi, le terrain est assurément un moment privilégié pour observer, pour décrire des situations, des pratiques, des activités. Mais il est aussi un moment privilégié pour nouer des relations de parole avec les membres du groupe étudié, des relations qu'on n'aurait pas forcément par la technique plus classique de l'entretien, qui sont possibles parce que l'on est inscrit, dans la durée, dans l'univers de vie des enquêtés et dans des relations relativement étroites avec eux. Le terrain est pour moi un moment privilégié pour faire de la sociologie compréhensive, un moment pour être wébérien. Et en même temps non, au sens où je serais plus attentif, aujourd'hui, à observer et décrire. Je ne le fais pas suffisamment dans le livre.

\section{Les sorties de terrain et la question de la restitution : naviguer entre différents impératifs}

I : Nous avons parlé des manières d'entrer et de se maintenir sur les terrains, mais qu'en est-il de la sortie, comment cela s'est passé pour vous, sur vos terrains?

os: Ça aussi c'est difficile. Bien difficile! Pour l'enquête sur le Nord, je n'avais pas le choix, parce que tout ça se passait dans le cadre d'une thèse, il fallait terminer cette thèse, même si j'ai vraiment bénéficié, de ce point de vue, de conditions institutionnelles bien plus permissives qu'aujourd'hui. Et puis je crois que j'étais moins exigeant vis-à-vis de moi-même, parce que c'était une période où l'enquête ethnographique était moins pratiquée qu'aujourd'hui chez les sociologues, le milieu était donc moins exigeant sur ce plan, mon « sur-moi » était donc moins exigeant lui aussi ! Pour l'enquête sur la RATP par contre, j'ai beaucoup plus de mal à finir, à sortir $\mathrm{du}$ « terrain »... Comme je ne suis plus sous pression institutionnelle, puisque je ne suis plus en activité, je n'ai pas de délai impératif à tenir - si ce n'est en termes d'âge, je ne suis plus tout jeune... Du coup, je suis beaucoup plus exposé au risque d'attendre d'avoir absolument tous les éléments que je souhaite pour pouvoir écrire. Et ça, c'est évidemment un piège redoutable! Alors je me bats contre cela en essayant de faire deux choses en même temps, c'est-à-dire rédiger les parties du livre que j'ai en tête, dont je suis à peu près certain, tout en continuant à enquêter sur les parties pour lesquelles j'estime que ce n'est pas au point. Mais de toute manière je suis pris dans une tension entre la nécessité, d'un côté, de savoir mettre un point final et écrire, et le fait, de l'autre, que pour qu'une enquête ait de l'intérêt, il ne suffit évidemment pas de décrire, il faut induire, interpréter, mettre en perspective, mais sans pour autant induire arbitrairement, n'importe comment, d'où la tentation de différer constamment les inductions tant qu'on n'a pas tous les éléments. Ce qui m'a retardé aussi, c'est que longtemps, très longtemps, je me suis dit : «Bon d'accord, voilà ce que je peux décrire. Mais qu'est-ce que je peux répondre à la question du "so what" ? » Ces deux raisons (la crainte de ne pas avoir des matériaux suffisamment robustes pour induire, et la crainte que ce que j'avais à dire ne soit pas vraiment intéressant) ont fait que, dans mon cas, s'agissant de l'enquête sur la RATP, la sortie du terrain a été considérablement longue (beaucoup trop, certainement, 
mais je n'ai pas su faire autrement) et retardée. Je suis seulement en train de sortir du terrain, pour passer à l'écriture. Je n'aurais jamais pu écrire Les ficelles du métier de Becker (2002)!...

I : La sortie du terrain est donc avant tout un enjeu scientifique. Que pensez-vous alors de cette évolution des contraintes formalisées, temporelles, statutaires... sur les enquêtes ethnographiques ? Parce que vous avez deux exemples d'enquêtes très longues en fait, qui sont un privilège...

os: Un très grand privilège, même ! Quoique l'enquête sur les conducteurs de bus ait été beaucoup trop longue, j'aurais dû poser un point final il y a un bon moment déjà. Mais je vois bien ce que vous voulez dire. Il y a une pression de plus en plus forte, depuis une dizaine d'années, sur les doctorants, en France, pour des thèses qui n'excèdent pas quatre à cinq ans. Et je ne pense pas grand bien de cette évolution. Pour l'enquête ethnographique, étant donné le temps que requiert l'entrée sur un terrain, la saisie de ce qui s'y passe, la construction d'un objet d'investigation, quatre ans pour une thèse, sachant qu'il est également exigé, pour avoir une chance d'être recruté comme maître de conférence, d'avoir, au cours de la période de la thèse, assumé des tâches d'enseignement, et d'avoir publié, c'est extrêmement difficile, voire impossible. Une telle pression, si elle devait s'accentuer encore (et c'est bien possible), conduirait tôt ou tard, purement et simplement, à un appauvrissement drastique des enquêtes ethnographiques menées dans le cadre des thèses. C'est-à-dire à un appauvrissement de la sociologie...

I: La sortie du terrain pose aussi directement ou indirectement la question de la restitution aux enquêté·ess. Pensez-vous que cette question se pose différemment aujourd'hui ?

os : Il me semble qu'elle se pose nécessairement différemment parce que les enquêtés ont aujourd'hui, beaucoup plus qu'auparavant, les moyens de savoir ce que l'on dit d'eux, par les médias, et par internet, par tout ce qui est aujourd'hui rendu accessible en ligne. Et c'est vrai même s'agissant des gens auxquels je m'intéresse, qui n'occupent pourtant pas des positions sociales spécialement élevées. J’ai plusieurs fois été frappé par ce que certains des conducteurs de bus que je connaissais étaient parvenus à savoir de moi, simplement par Google et par Wikipédia. D'où la nécessité, plus que jamais, de se préoccuper de l'image que renvoie ce que l'on écrit de ceux qui en sont l'objet. En ce qui me concerne par exemple, il y a d'ores et déjà, s'agissant de la RATP, des choses dont je sais qu'il me sera difficile de les dire. Concernant le conducteur de bus « de base », là il n'y a pas trop problème, parce qu'ils sont 15 000, ce que m'a dit tel conducteur un jour sur telle ligne, je peux le reprendre et l'anonymiser de manière à ce que nul ne puisse le reconnaître. Mais c'est beaucoup plus délicat quand il s'agit de responsables syndicaux, qui m'ont livré, consciemment ou inconsciemment, des choses, des aspects, dans certains cas importants, de leur univers moral et idéologique. Là il me sera beaucoup plus difficile d'en parler car ils sont davantage identifiables, et je ne veux en aucun cas leur nuire. Là se posent de vraies questions déontologiques.

Pour en venir, justement, à ces questions de manière plus générale, je ne me sens vraiment pas en possession de réponses les concernant tant ces sujets sont difficiles. Il y a simplement deux choses auxquelles je crois. La première est qu'il y a un droit, pour l'ethnographe, à la non-transparence dans les rapports avec ses enquêtés, un droit à ne pas tout dire de ses objectifs, à dissimuler certaines stratégies, à une certaine part, pour reprendre une expression de Daniel Céfaï, de « double jeu » (Cefaï et Amiraux, 2002). Je crois qu'il faut assumer et défendre ce droit, je ne vois pas comment certaines enquêtes seraient possibles sans cela. Je suis 
très opposé de ce point de vue, comme beaucoup de collègues français, à cette prolifération à laquelle on assiste dans le monde nord-américain des protocoles, des contrats, des comités d'éthique. Nous avons la chance, pour l'instant en France, de ne pas avoir cela. Et en même temps, second repère tout aussi essentiel, nous avons évidemment l'obligation absolue, en tant qu'ethnographes, de nous préoccuper de ce que nos publications peuvent « faire » à nos enquêtés, tant parce que d'autres peuvent les reconnaître que parce qu'eux-mêmes peuvent s'y reconnaître. L'ethnographie perdrait toute légitimité si celui qui la pratique perdait de vue cette exigence, ce souci de ce qu'il doit à ses enquêtés, à ceux qui lui ont accordé leur confiance, ou s'il s'en affranchissait. Pas d'obligation de transparence dans l'enquête, mais une obligation d'éthique dans l'écriture et la publication. Cela étant, une fois dites ces deux choses, les difficultés, dans mon expérience tout au moins, ne font souvent que commencer. Où finit le droit à une certaine non-transparence, où commence la tromperie, l'instrumentalisation? S'agissant de l'écriture et de la publication, le refus que celles-ci puissent constituer une forme de violence pour ceux dont on parle est une chose, mais jusqu'où aller dans le souci des effets potentiels de ce que l'on écrit pour ceux qui en sont l'objet? Faut-il inconditionnellement vouloir préserver l'image des enquêtés pour eux-mêmes et pour d'autres ? Est-il possible d'écrire avec cette injonction? Quelles limites se donner si on la transgresse? Bref, qu'on enquête ou qu'on écrive, comment faire lorsqu'on est aux limites du «moral » ? Ces questions m'ont toujours accompagné, et je sais d'avance que je les retrouverai dans la rédaction de certaines parties de mon enquête sur les conducteurs.

I : Cela nous amène à une dernière question sur la culpabilité... C’est un tabou en sociologie...

os : Oui.

$\mathrm{I}$ : Pourtant cette question est assez transversale, et nous l'avons tous et toutes éprouvée à un moment donné ou à un autre, soit à la parution d'un livre ou d'un article, soit simplement en sortant d'un entretien...

os: Oui, et nous manquons sans doute de lieux d'échange pour confronter les perceptions de ces questions, et les réponses que les uns et les autres leur apportent.

I : Oui, les lieux d'échange sont primordiaux... car nous pensons aussi que les sociologues sont très peu armés pour réfléchir à cela...

os : Je suis d'accord avec vous. C'est quelque chose, effectivement, qui est peu dit, je crois parce que les sociologues se méfient souvent des questions morales. C'est-à-dire qu'ils considèrent que voilà, c'est...

I : Que cela n'est pas de leur ressort?

os : Peut-être pas, mais je pense que certains se disent : «Si je sociologise la situation, si je raisonne en sociologue, le problème perdra son aspect "moral".Je m'apercevrai que chacun, enquêteur et enquêté, investit certains intérêts sociaux dans l'enquête. C'est tout. Il n'y a ni bon ni méchant, personne, dans cette affaire, n'est moral ou immoral ». Je comprends l'idée. Mais je ne suis pas convaincu qu'il soit toujours possible de se sortir ainsi de la difficulté. Dans mon livre sur le Nord, j'avais consulté deux de mes enquêtés pour savoir s'ils étaient d'accord pour que je parle d'eux dans le livre. Ce ne sont pas les quatre personnages du chapitre final, ce sont ceux que j'appelle André et Marie-Line « Mounier». Lui - il était ouvrier qualifié en usine - avait aménagé, au premier étage de son pavillon, une sorte de bureau, dans lequel il se livrait à diverses occupations. En prévision d'achats qu'il projetait de faire (objets pour la maison, outils ou matériaux pour du bricolage...), il y rassemblait des catalogues, qu'il passait beaucoup de temps à lire, et dont il extrayait 
des informations qu'il notait, rangeait, classait dans des cahiers. Et il m'avait semblé qu'il y avait, dans cet espace qu'il s'était créé, l'expression intense d'une aspiration sociale, aspiration à être, en dehors de l'usine, quelqu'un d'autre qu'un ouvrier... J'ai donc décrit cet espace en ces termes dans le livre. Quand celui-ci est sorti, je le leur ai envoyé, à lui et Marie-Line. Et ça s'est très mal passé. Je les ai blessés l'un et l'autre, mais surtout lui, dans la manière dont j'ai parlé de ce bureau. Je lui ai donné le sentiment que je me moquais de lui, que je le percevais comme l'ouvrier qui se voit plus haut que ce qu'il est... Lorsque j'ai téléphoné, le livre étant sorti (comme je le leur avais envoyé, je souhaitais savoir s'ils l'avaient bien reçu, si je pouvais venir les voir), c'est son épouse qui m'a répondu, qui m’a dit qu'il avait très mal vécu la manière dont je parlais de lui.J'ai alors demandé si je pouvais néanmoins venir. Elle m’a répondu que lui me faisait dire que c'était d'accord pour que je vienne, mais que, «si c'était pour me moquer d'eux à nouveau, ce n'était pas la peine ». Je suis venu quand même. Nous avons passé une soirée difficile, et je ne les ai plus revus ensuite. Ça a été... ça ne s'est pas réparé. Pourquoi vous raconter cela ? Parce qu'il n'y a pas trente-six manières de caractériser mon erreur à ce moment-là. J'ai méconnu, dans ce passage du livre, les effets que celui-ci risquait d'entraîner pour lui, le potentiel de violence qu'il comportait. Je l'ai blessé. Y avait-il une autre manière possible d'écrire ce passage ? Ou fallait-il s'interdire purement et simplement la présentation de cet exemple? Je n'ai pas la réponse. Mais quelle qu'elle soit, il y a eu faute de ma part, et cela d'autant plus que l'un et l'autre m'avaient clairement ouvert leurs portes pendant l'enquête.

I : Et cette culpabilité vous hante encore?

os : Non, ce serait bien entendu faux de le dire, parce que tout cela remonte maintenant à plus de trente ans. Mais ce que tout cela m'a fait comprendre, c'est qu'il peut y avoir faute morale dans un texte d'ethnographie, que l'écriture ethnographique doit comporter une attention aux questions éthiques, et que même si personne ne peut se prétendre en possession de quelque certitude que ce soit sur les limites de ce que l'on peut s'autoriser à écrire, il faut toujours rester attentif à cette question. Et j'espère bien que je saurai, cette fois, ne pas l'oublier dans le livre sur les conducteurs... 


\section{Bibliographie}

BECKER Howard, 2002, Les ficelles du métier. Comment conduire sa recherche en sciences sociales, Paris, La Découverte, 360 p.

Burawoy Michael, 1998, « The Extended Case Method », Sociological Theory, vol. 16, $\mathrm{n}^{\circ} 1$, p. 4-33.

Cefaï Daniel \& Amiraux Valérie, 2002 « Les risques du métier. Engagements problématiques en sciences sociales. Partie 1 », Cultures \& Conflits, vol. $3, \mathrm{n}^{\circ} 47$, p. 15-48.

DARGÈRE Christophe, 2012, L'observation incognito en sociologie. Notions théoriques, démarche réflexive, approche pratique et exemple concrets, Paris, L'Harmattan, 170 p.

HoggaRT Richard, 1970, La Culture du pauvre, Étude sur le style de vie des classes populaires en Angleterre, Paris, Éditions de Minuit, 424 p.

RENAHY Nicolas, 2016, « L'ethnographie comme expérience de conversion à la sociologie. Entretien avec Olivier Schwartz. », Cahiers de recherche sociologique, $\mathrm{n}^{\circ}$ 61, p. 191-215.

SchWARTz Olivier, 1990, Le monde privé des ouvriers. Hommes et femmes du Nord, Paris, Presses Universitaires de France, 544 p.

SCHWARTZ Olivier, 1993, « L'empirisme irréductible », postface à ANDERSON Nels, Le Hobo, sociologie du sans-abri, Paris, Armand Colin, p. 265308. 